CLINICAL STUDY

\title{
Pulsatile secretory characteristics of allopregnanolone, a neuroactive steroid, during the menstrual cycle and in amenorrheic subjects
}

Alessandro D Genazzani, Michele Luisi ${ }^{1}$, Barbara Malavasi, Claudia Strucchi, Stefano Luisi ${ }^{1}$, Elena Casarosa ${ }^{1}$, Francesca Bernardi ${ }^{1}$, Andrea R Genazzani ${ }^{1}$ and Felice Petraglia ${ }^{2}$

Department of Obstetrics and Gynecology, University of Modena, ${ }^{1}$ Department of Obstetrics and Gynecology, University of Pisa and ${ }^{2}$ Department of Obstetrics and Gynecology, University of Siena, Italy

(Correspondence should be addressed to Alessandro D Genazzani, Clinica Ostetrica Ginecologica, Università di Modena, Via del Pozzo 71, 41100 Modena, Italy; Email: algen@unimo.it)

\begin{abstract}
Objective: To investigate whether allopregnanolone, a neuroactive steroid involved in modulating behavioural and neuroendocrine functions, shows episodic secretion in eumenorrheic women, during the follicular and luteal phases of the menstrual cycle, and in women with stress-induced amenorrhea.

Patients: Six eumenorrheic women and 14 women with hypothalamic amenorrhea were enrolled for the present study.

Methods: All subjects underwent hormonal evaluation in baseline conditions and a pulsatility study to determine LH, cortisol and allopregnanolone episodic release. Eumenorrheic subjects were investigated twice, in the follicular phase (days 3-7) and in the luteal phase (days 18-22) of the menstrual cycle. LH, FSH, prolactin, estradiol, phosphate, DHEA, allopregnanolone and cortisol levels were evaluated in each case.

Results: In healthy women, serum gonadotropin and gonadal steroid levels were significantly lower $(P<0.01$ and $P<0.05$ respectively) than those in amenorrheic subjects. Allopregnanolone was higher in amenorrheic subjects and during the luteal phase, compared with the follicular phase, of eumenorrheic subjects $(P<0.01)$. Pulse analysis revealed a significant episodic discharge of allopregnanolone in all subjects (follicular phase $6.5 \pm 0.3$ peaks $/ 6 \mathrm{~h}$ and luteal phase $5.5 \pm 0.4$ peaks $/ 6 \mathrm{~h}$, hypothalamic amenorrhea $7.0 \pm 0.7$ peaks $/ 6 \mathrm{~h}$ ) with higher pulse amplitude in amenorrheic subjects and during the luteal phase compared with the follicular phase of the eumenorrheic subjects $(P<0.05)$. Moreover, the specific concordance index demonstrated that allopregnanolone is coupled with LH only during the luteal phase of the cycle and with cortisol during both phases. Allopregnanolone-cortisol coupling was also observed in amenorrheic subjects.

Conclusions: Allopregnanolone is secreted episodically. Both the ovary and adrenal glands release this steroid hormone and it shows temporal coupling with LH only during the luteal phase, with cortisol during both the studied phases of the menstrual cycle in eumenorrheic women and again with cortisol in hypothalamic amenorrheic patients.
\end{abstract}

European Journal of Endocrinology 146 347-356

\section{Introduction}

Neurosteroids modulate behavioural functions and neuroendocrine axes in experimental animals $(1-3)$, most probably acting via the $\gamma$-aminobutyric $\operatorname{acid}_{A}$ $\left(\mathrm{GABA}_{\mathrm{A}}\right)$ receptors $(4,5)$, and they are considered the most potent endogenous antidepressant substances (1-3). Neurosteroids, such as allopregnanolone, are synthesised in the brain, either de novo from cholesterol or by the local metabolism of blood-borne precursors (6). Studies in humans have clearly demonstrated that their plasma concentrations are very dependent on gender and age $(1-3,7)$. Studies in rats and humans have further clarified that allopregnanolone, the most important and active among the neurosteroids, is produced by the ovary, increases and decreases during the ovulatory cycle but does not disappear after ovariectomy $(7,8)$. In fact, the adrenal glands are another important source of neurosteroids, primarily allopregnanolone, as only the combination of ovariectomy and adrenalectomy in rats almost completely eliminates this neurosteroid from the circulation (8). 
Recently, studies on circulating allopregnanolone have been carried out in humans to try to reveal some of its characteristics. The concentrations of allopregnanolone seem to be relevant since this hormone has been demonstrated to be involved in the modulation of stress, mood and sexual behaviour in rats $(9-12)$ and is probably similarly involved in humans. In fact, allopregnanolone production has recently been demonstrated to be reduced in women suffering from premenstrual syndrome (PMS) thus indicating that allopregnanolone is relevant in the modulation of human neuro/psycho/endocrine functions also (13). In addition, it has been observed that serum allopregnanolone levels change throughout the menstrual cycle, with the highest levels during the luteal phase (7, 14 ), thus supporting the hypothesis of a common pathway for allopregnanolone and progesterone secretion in humans. The adrenal glands are also involved in allopregnanolone production $(7,8)$ since both corticotrophin-releasing factor (CRF) and adrenocorticotrophin (ACTH) stimulation tests in humans are able to increase allopregnanolone plasma levels within 3060 min (7). Since all steroids secreted from endocrine glands show specific pulsatile release, it is possible that allopregnanolone might also show episodic release in view of its adrenal and/or ovarian origins.

On the basis of the neuro/psycho/endocrine relevance of allopregnanolone, the present study aimed to verify the secretory characteristics of allopregnanolone in healthy subjects during the follicular and luteal phases of the menstrual cycle and in patients with hypothalamic amenorrhea. The latter were taken as models of stress-induced neuroendocrine dysfunction (15-17). In addition, luteinizing hormone (LH) and cortisol pulsatile release were evaluated in order to define the temporal/functional linkage between allopregnanolone and the gonadotrophin-releasing hormone (GnRH)-gonadotrophin or ACTH-cortisol axes.

\section{Material and methods}

\section{Subjects}

After giving their informed consent, 20 women were included in the present study. They were subdivided into two groups, one of fertile women $(n=6)$, aged 24-29 years, and a second of patients with hypothalamic amenorrhea $(n=14)$, aged $19-27$ years.

Healthy subjects were enrolled according to the following criteria: a) a history of normal menstrual cycles (28-32 days) during the last 12 months, b) no endocrine disease and normal thyroid and adrenal function and c) no consumption of oral contraceptives.

Criteria for inclusion of amenorrheic patients were: a) the absence of menses for at least 6 months before the study, b) no evidence of pregnancy, c) the absence of metabolic or endocrine diseases, d) normal prolactin (PRL) and thyroid hormone levels, e) hypogonadotrophic condition ( $\mathrm{LH}<3 \mathrm{mIU} / \mathrm{ml}$ ), f) body weight within the normal ranges for age and height, i.e. a body mass index (BMI) not below $19 \mathrm{~kg} / \mathrm{m}^{2}$, g) no history of emotionally stressful events preceding the onset of amenorrhea, such as problems within the family, at school, at work or of psycho-social stress. Psychiatric diseases were excluded using DSM-IV criteria (18).

Informed consent was obtained from each subject after description of the protocol approved by the local Ethics Committee of the University of Pisa.

\section{Study protocol}

Hormonal parameters (LH, follicle stimulating hormone (FSH), PRL, oestradiol (E2), progesterone (P), DHEA and cortisol) were evaluated in all subjects at baseline conditions. In addition, both groups of subjects underwent frequent sampling (in the pulsatility study) to assess the spontaneous episodic secretion of LH, cortisol and allopregnanolone. Fertile women undertook this study twice, once during the follicular phase (days $3-7$ ) and once during the luteal phase (days 18-22) of the menstrual cycle. The pulsatility study was performed by inserting a heparin well in an antecubital vein 45-60 min before commencing venous sampling and blood was withdrawn every $10 \mathrm{~min}$ for $6 \mathrm{~h}$ from $0830 \mathrm{~h}$ to $1430 \mathrm{~h}$.

\section{Assays}

All samples from the same subject were assayed in duplicate in each assay. Basal LH and FSH concentrations were determined using an immunofluorimetric assay, as described previously $(19,20)$. Basal PRL, E2, P, DHEA and cortisol concentrations were determined using a commercially available radioimmunoassay (Radim RIA; Pomezia, Rome, Italy) whose intra-assay and interassay coefficients of variation ranged between $4.1 \%$ and $8.8 \%$, as previously described $(20,21)$.

Allopregnanolone concentrations were determined, after ether extraction and Sep-Pak (Waters, Milan, Italy) partition, using an RIA method described previously (7). The polyclonal antiserum raised against allopregnanolone was kindly provided by $\mathrm{Dr} \mathrm{R} \mathrm{H}$ Purdy. The sensitivity of the assay was $20 \mathrm{pg} /$ tube (minimal detectable dose) and the intra- and interassay coefficients of variation were $7.0 \%$ and $9.0 \%$ respectively (7).

\section{Pulse detection}

Time series of LH, cortisol and allopregnanolone were first evaluated separately to estimate the random measurement error on the duplicates (using the program PREDETEC.WK1; $(22,23)$ ) then secretory episodes for each hormone time series were identified using the program DEтECT $(22-24)$ with a $P$ value equal to $0.01(1 \%)$ for the nominal false positive rate. At the 
nominal $P$ level of 0.01 ( $1 \%$ ) for false positive errors, DETECT showed a consistent specificity, as previously reported (24), since the false positive rate observed in the data of the plasma pool of each subject assayed together with the time series was not statistically different from $1 \%$.

\section{Estimation of the degree of concordance}

The presence of any significant concomitance between the secretory events of LH and allopregnanolone, or cortisol and allopregnanolone, was assessed by computing the specific concordance (SC) index (25), interposing various lags between each time series couplet under analysis. Time series of each hormone (A and B) were converted into 'quantized' time series, where only the first sample (the onset) of each detected peak was taken to represent the occurrence of that peak (25). These quantized data series were then matched, and quantized values for hormone A were compared with the corresponding values for hormone B. The presence or absence of an event (an onset) in either or both series is counted and then SC index is computed. A spectrum of SC values can be constructed by sliding the two series, interposing integral multiples of the sampling interval as the lag. The presence of a positive lag time indicates that an event in A preceded the secretion event in B.

SC spectra were evaluated for each subject and the mean SCs over each group of patients, at each lag time, were calculated, thus obtaining a mean SC spectrum. The location of the maximum of the mean SCs for each group of women was also noted. Monte Carlo simulations were then performed to study the frequency distribution of the SC index under the null hypothesis of random concordance, as previously described $(25,26)$. For each pair of clinical data (two pairs for each subject), 500 simulated pairs of simulated series were created and frequency distributions of SC obtained. The observation of a SC value above the $95 \%$ percentile of the frequency distribution generated for that individual, or for the whole group of subjects, allowed rejection of the hypothesis of random coincidence at the $P<0.05$ confidence level (25).

\section{Statistical analysis}

The presence of significant differences between groups was tested, after analysis of variance (one-way ANOVA), using Student's t-test for paired and unpaired data as appropriate. Linear regression analysis was used to test the correlations between individual hormonal values.

\section{Results}

The hormonal characteristics of the 2 groups of subjects are reported in Table 1 as means \pm S.E.M. Control subjects showed typical hormonal changes related to the phases of the menstrual cycle and, as expected, DHEA and allopregnanolone levels were significantly higher during the luteal compared with the follicular phase of the cycle. Amenorrheic women showed significantly lower LH, E2 and P plasma levels $(P<0.01)$ and higher allopregnanolone plasma levels throughout, similar to controls during the luteal phase. Cortisol plasma levels were higher in amenorrheic subjects $(P<$ 0.01) compared with the controls during both phases.

The characteristics of the episodic secretion of LH, cortisol and allopregnanolone of both groups of subjects are reported in Table 2 (LH, cortisol and allopregnanolone levels were expressed as the integrated mean of the pulsatility). Fig. 1 shows the typical secretory profiles of LH, cortisol and allopregnanolone in control subjects and Fig. 2 shows the same in amenorrheic subjects. All three examined hormones showed a distinct episodic release, in both the controls and

Table 1 Hormonal characteristics of patients under study (means \pm S.E.M.).

\begin{tabular}{lccc}
\hline & \multicolumn{2}{c}{ Controls $(n=6)$} & $\begin{array}{c}\text { Hypothalamic amenorrhea } \\
(n=14)\end{array}$ \\
\cline { 2 - 3 } & Follicular phase & Luteal phase & $\begin{array}{c}\text { ame } \\
\text { LH }(\mathrm{mlU} / \mathrm{ml})\end{array}$ \\
FSH $(\mathrm{mlU} / \mathrm{ml})$ & $2.2 \pm 0.3$ & $4.0 \pm 0.2^{*}$ & $2.8 \pm 0.2 \S$ \\
$\mathrm{PRL}(\mathrm{ng} / \mathrm{ml})$ & $3.1 \pm 0.3$ & $3.8 \pm 0.3$ & $4.1 \pm 0.7 \S$ \\
$\mathrm{E} 2(\mathrm{pg} / \mathrm{ml})$ & $8.2 \pm 1.1$ & $11.8 \pm 1.7$ & $15.2 \pm 2.4 \S$ \\
$\mathrm{P}(\mathrm{ng} / \mathrm{ml})$ & $35.4 \pm 3.0$ & $95.8 \pm 11.3 \S$ & $0.3 \pm 0.1$ \\
DHEA $(\mathrm{ng} / \mathrm{dl})$ & $0.4 \pm 0.1$ & $7.7 \pm 1.2 \S$ & $340.2 \pm 16.8$ \\
Cortisol $(\mu \mathrm{g} / \mathrm{l})$ & $260.5 \pm 13.5$ & $830.2 \pm 31.3 \S$ & $205.2 \pm 10.1^{*}$ \\
Allopregnanolone $(\mathrm{pg} / \mathrm{ml})$ & $124.2 \pm 15.1$ & $135.2 \pm 12.5$ & $600 \pm 12.6^{* *}$ \\
BMl $\left(\mathrm{kg} / \mathrm{m}^{2}\right)$ & $240.7 \pm 11.5$ & $715.2 \pm 24.3^{* *}$ & $20.4 \pm 0.2 \S$ \\
\hline
\end{tabular}

${ }^{\star} P<0.05$ vs follicular and luteal phases, ${ }^{* \star} P<0.01$ vs follicular phase, and $\S P<0.01$ vs follicular and luteal phases.

Conversion factor to SI units for LH and $\mathrm{FSH}: \mathrm{mlU} / \mathrm{ml}$ to IU/l is 1 , prolactin (PRL): $\mathrm{ng} / \mathrm{ml}$ to $\mu \mathrm{g} / \mathrm{l}$ is 1 , oestradiol (E2): $\mathrm{pg} / \mathrm{ml}$ to $\mathrm{pmol} / \mathrm{l}$ is 3.699 , progesterone $(P): \mathrm{ng} / \mathrm{ml}$ to $\mathrm{nmol} / \mathrm{l}$ is 3.18 , DHEA: $\mathrm{ng} / \mathrm{dl}$ to $\mathrm{nmol} / \mathrm{l}$ is 0.0347 , cortisol: $\mu \mathrm{g} / \mathrm{l}$ to $\mathrm{nmol} / \mathrm{l}$ is 2.579 , and allopregnanolone: $\mathrm{pg} / \mathrm{ml}$ to $\mathrm{nmol} / \mathrm{l}$ is 0.003185 . 
Table 2 Characteristics of spontaneous episodic release of LH, cortisol and allopregnanolone of the subjects under study (means \pm S.E.M.).

\begin{tabular}{|c|c|c|c|c|c|c|c|c|c|}
\hline & \multicolumn{3}{|c|}{ LH (mlU/ml) } & \multicolumn{3}{|c|}{ Cortisol ( $\mu \mathrm{gl})$} & \multicolumn{3}{|c|}{ Allopregnanolone (pg/ml) } \\
\hline & mean $^{+}$ & peaks/6h & amplitude & mean $^{+}$ & peaks/6h & amplitude & mean $^{+}$ & peaks/6h & amplitude \\
\hline Follicular phase & $2.7 \pm 0.2$ & $3.5 \pm 0.4$ & $0.85 \pm 0.2$ & $134.6 \pm 8.8$ & $6.5 \pm 0.3$ & $65.7 \pm 8.2$ & $260.6 \pm 10.4$ & $6.5 \pm 0.3$ & $138.6 \pm 8.1$ \\
\hline Luteal phase & $4.5 \pm 0.4$ & $5.5 \pm 0.5$ & $2.1 \pm 0.4^{\star \star}$ & $122.4 \pm 10.1$ & $6.5 \pm 0.4$ & $50.5 \pm 7.5$ & $743.6 \pm 13.4^{\star *}$ & $5.5 \pm 0.4$ & $315.3 \pm 13.0^{*}$ \\
\hline Amenorrhea & $1.2 \pm 0.3$ & $8.0 \pm 0.3^{*}$ & $0.4 \pm 0.1 \S$ & $190.9 \pm 12.6^{*}$ & $8.2 \pm 0.7$ & $46.8 \pm 5.3$ & $615.8 \pm 10.5^{\star \star}$ & $7.0 \pm 0.7$ & $332 \pm 11.3^{*}$ \\
\hline
\end{tabular}

${ }^{+}$Means are the integrated means of pulsatilities.

${ }^{*} P<0.05$ vs follicular phase, ${ }^{* *} P<0.01$ vs follicular phase and $\S P<0.001$ vs follicular and luteal phases.

Conversion factor to $\mathrm{SI}$ units for $\mathrm{LH}: \mathrm{mIU} / \mathrm{ml}$ to $\mathrm{IU} / \mathrm{l}$ is 1 , cortisol: $\mu \mathrm{g} / \mathrm{l}$ to $\mathrm{nmol} / \mathrm{l}$ is 2.579 , and allopregnanolone: $\mathrm{pg} / \mathrm{ml}$ to $\mathrm{nmol} / \mathrm{l}$ is 0.003185 .

amenorrheic subjects. As for the pulsatile characteristics (i.e. pulse frequency and amplitude), LH pulse amplitude was significantly higher during the luteal than during the follicular phase $(P<0.01)$, and it was lowest in amenorrheic patients $(P<0.01)$ (Table 2). Conversely, LH pulse frequency was higher in amenorrheic patients than in control subjects.

Regarding cortisol pulsatile release, no differences were observed in either pulse amplitude or frequency, while plasma levels were higher in amenorrheic than in control subjects. Allopregnanolone showed significant episodic discharge in all subjects. Control subjects presented higher concentrations and a higher pulse amplitude during the luteal than during the follicular phase. Amenorrheic patients showed plasma allopregnanolone levels and pulse amplitudes similar to the luteal phases but higher than those of the follicular phases $(P<0.01$ and $P<0.05$ respectively) of the control subjects (Table 2). No significant correlations were observed between the concentrations and pulsatile characteristics of LH and cortisol and those of allopregnanolone in either of the groups under study.

When the SC index was computed, LH and allopregnanolone were temporally coupled at time 0 (i.e. after the onset of each LH pulse, a pulse of allopregnanolone followed within 0-10 min) during the luteal but not during the follicular phase of the menstrual cycle (Fig. 3). On the other hand, cortisol and allopregnanolone were temporally coupled during both the luteal and follicular phases, with a significant SC index $(P<$ 0.05 ) at time lag +30 and $+70 /+80$ min (Fig. 4). These data indicate that each allopregnanolone pulse followed a cortisol pulse after a precise interval of time. The presence of two distinct significant concordant time intervals over the explored range indicates that allopregnanolone pulses probably have a quite constant periodicity (every $30-40 \mathrm{~min}$ ) so as to appear synchronised more than once with the same cortisol pulse. Interestingly, amenorrheic subjects also showed a similar pattern of concordance between cortisol and allopregnanolone (Fig. 5) while no concordance was observed in these patients between LH and allopregnanolone (data not shown).

\section{Discussion}

The present study demonstrates for the first time that allopregnanolone has a spontaneous episodic discharge in healthy women during the menstrual cycle as well as in patients suffering from hypothalamic amenorrhea. In addition, our data support the fact that both the gonads and adrenal glands secrete allopregnanolone, although in an independent manner.

In humans, serum allopregnanolone levels change with the phase of the menstrual cycle and decrease with aging. In fact, the high levels during the luteal phase $(7,13,14)$ are strictly dependent on corpus luteum production of progesterone and its derivatives $(3,7)$. On the contrary, aging seems to induce a reduction in allopregnanolone plasma levels due to the menopause-induced failure of ovarian steroidogenesis, leaving the adrenal as the only source of allopregnanolone $(7,12)$.

Our present data confirm these observations as demonstrated by the consistent increase in allopregnanolone plasma levels during the luteal phase. In addition, the use of a highly sensitive and specific pulse-detector algorithm (24) allowed us to show for the first time that allopregnanolone has an episodic pulsatile release. During both phases of the menstrual cycle studied, a distinct, spontaneous pulsatile discharge of allopregnanolone was clearly evident and characterised by an apparent (i.e. relative to the sampling paradigm applied) pulse frequency and amplitude. The mean pulse frequency was similar during both the follicular and the luteal phases but the mean amplitude differed, presenting higher during the luteal phase. When allopregnanolone secretion was investigated in patients with hypogonadotropinemic hypothalamic amenorrhea, levels were higher than during the follicular phase but similar to the luteal phase of eumenorrheic subjects. In addition, amenorrheic patients showed a distinct episodic discharge of allopregnanolone similar in frequency to that of eumenorrheic subjects. As P levels are close to zero in hypothalamic amenorrhea due to the functional blockage of the ovaries, the adrenal glands remain the only 


\section{FOLLICULAR PHASE}

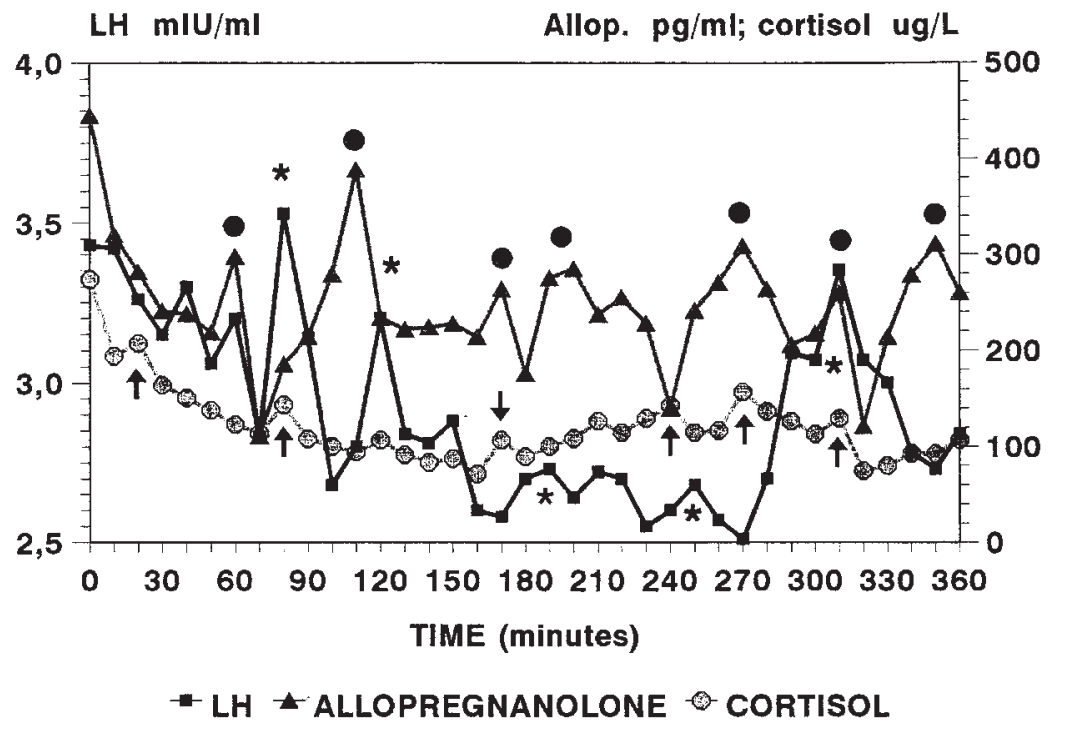

\section{LUTEAL PHASE}

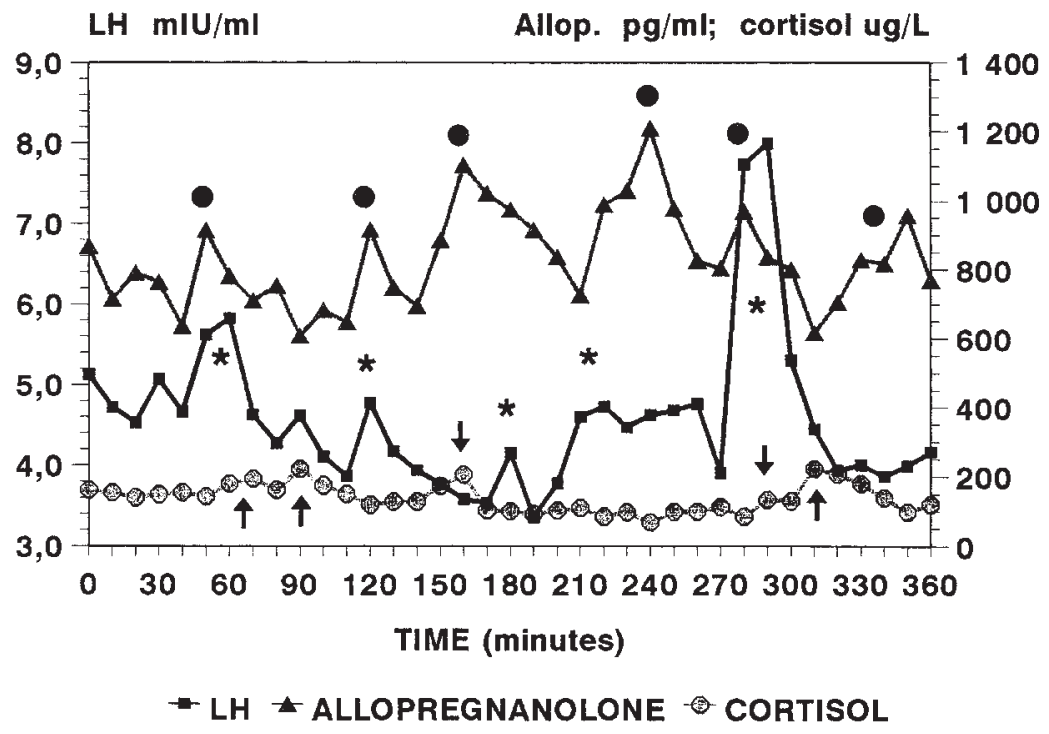

Figure 1 Secretory profiles for LH (ם), allopregnanolone $(\mathbf{\Lambda})$ and cortisol $(\bullet)$ during the follicular (upper panel) and luteal phases (lower panel) of the menstrual cycle in one healthy subject. Asterisks, solid circles and arrows indicate significant pulses of $\mathrm{LH}$, allopregnanolone and cortisol respectively, as found by the DETECT program. Conversion factor to SI units for $\mathrm{LH}: \mathrm{mlU} / \mathrm{ml}$ to $\mathrm{IU} / \mathrm{l}$ is 1 , cortisol: $\mu \mathrm{g} / \mathrm{l}$ to $\mathrm{nmol} / \mathrm{l}$ is 2.579 , and allopregnanolone: $\mathrm{pg} / \mathrm{l}$ to $\mathrm{nmol} / \mathrm{l}$ is 0.003185 . source of allopregnanolone in these patients. Indeed, since hypothalamic amenorrhea is normally characterised by a specific stress-induced increase in adrenal function $(27,28)$, our data sustain the hypothesis that the adrenal glands are the major source of allopregnanolone in stress-induced amenorrhea.

To verify whether or not the ovary and/or adrenal glands are the major sources of allopregnanolone secretion, we tested the temporal coupling of both LH and cortisol with allopregnanolone. The coupling between LH and allopregnanolone was significant only during the luteal but not during the follicular phase of the menstrual cycle. Interestingly, such significant coupling of LH and allopregnanolone pulses was found at time 0 (i.e. within $0-10 \mathrm{~min}$, the sampling paradigm being every $10 \mathrm{~min}$ ); very similar to what 
LH $\mathrm{mlU} / \mathrm{ml}$

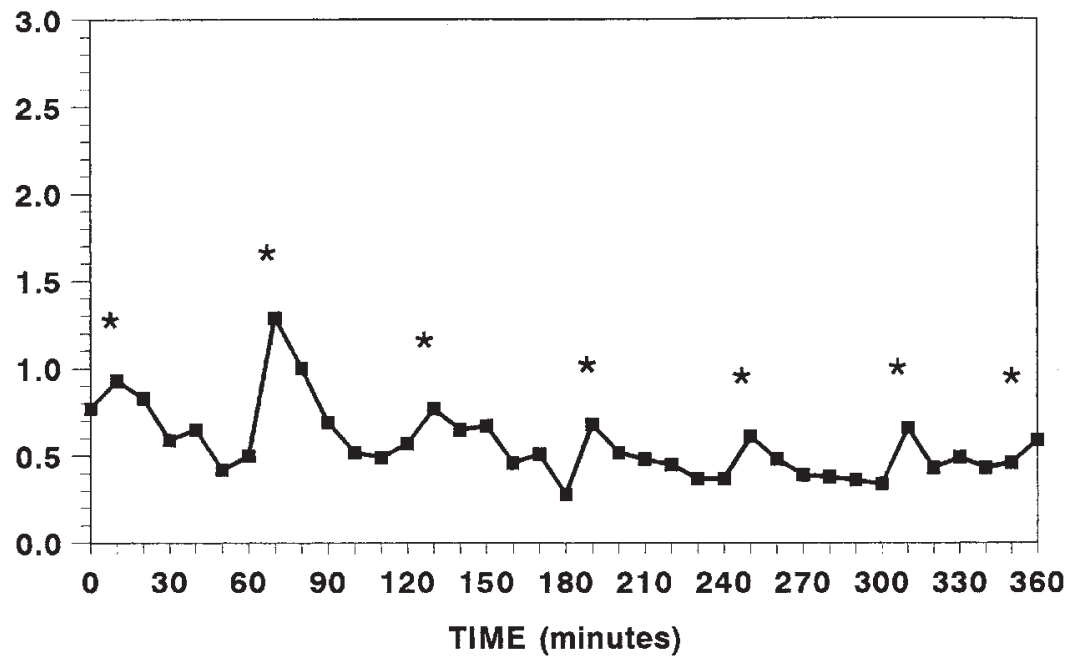

Figure 2 Secretory profile for LH ( $\square)$ in one amenorrheic patient. LH pulse frequency was higher than in control subjects but with lower amplitude. Asterisks indicate significant pulses of LH as found by the DETECT program. had already been observed with P (29). These results clearly suggest that, when ovarian production of $\mathrm{P}$ is present, LH is a driver for allopregnanolone secretion. Such a hypothesis is strongly supported by previous data showing that $\mathrm{LH}$ and $\mathrm{P}$ have perfectly similar temporal coupling (29) and by the fact that no temporal coupling was observed in amenorrheic patients, since no ovarian activity (i.e. no follicular development and ovulation) was present.

Of greater interest is the observation that allopregnanolone was temporally coupled to cortisol in both groups of subjects. This clearly supports the evidence of a specific adrenal role in producing allopregnanolone as an intermediate metabolite of adrenal steroid synthesis (30). These data are reinforced by the fact that amenorrheic patients presented plasma allopregnanolone levels similar to control subjects in the absence of normal ovarian function. In addition, the present study showed a temporal coupling of cortisol with allopregnanolone (with a time lag of $+30 /+40$ and $+70 /+80 \mathrm{~min}$ ), not only in both menstrual cycle phases of the control subjects, but also in the patients with hypothalamic amenorrhea, who have a functionally blocked ovary and a hyperactive adrenal gland. This finding is in agreement with previous reports $(23,29)$. The different coupling times between the LH (at time 0) and cortisol pulses (at time $+30 /+40$ ) with the allopregnanolone pulses is clear evidence of the differing responses of ovarian and adrenal tissues to their specific endocrine-stimulating signals (i.e. $\mathrm{GnRH}-$ LH and CRF-ACTH axes). Such different time lags might also be due to a different post-receptor signal transduction as well as to different enzymatic pathways involved in and/or modulating allopregnanolone

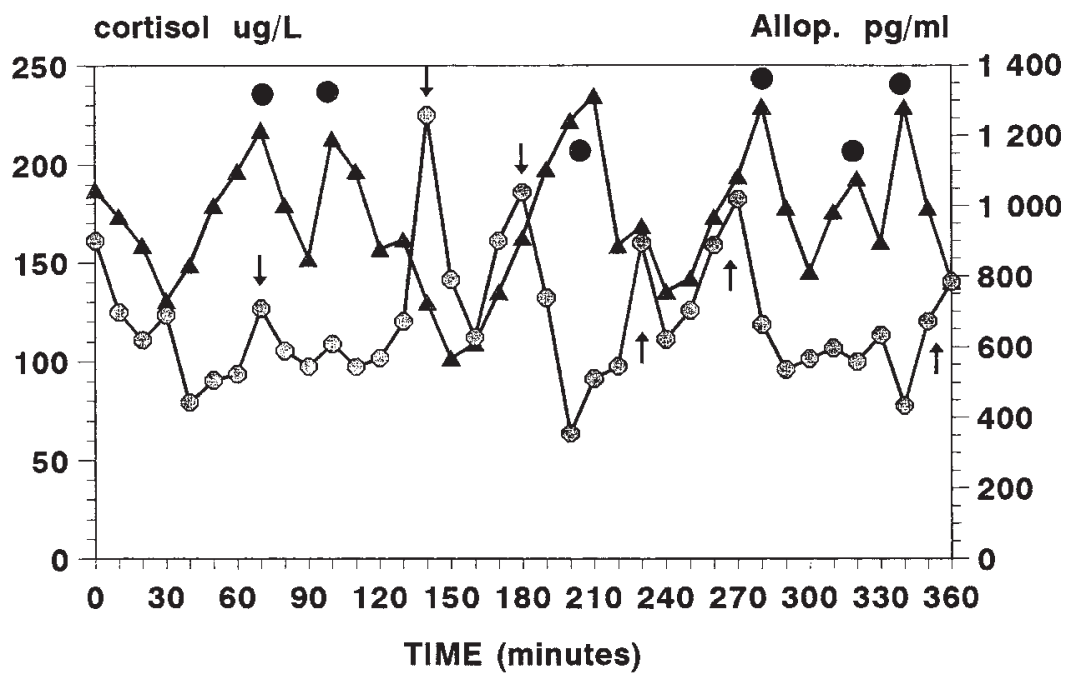

Figure 3 Secretory profiles for allopregnanolone $(\boldsymbol{\Lambda})$ and cortisol $(\bullet)$ in one amenorrheic subject. Solid circles and arrows indicate significant pulses of allopregnanolone and cortisol respectively, as found by the DETECT program. Conversion factor to SI units for cortisol: $\mu \mathrm{g} / \mathrm{l}$ to $\mathrm{nmol} / \mathrm{l}$ is 2.579 and allopregnanolone: $\mathrm{pg} / \mathrm{l}$ to $\mathrm{nmol} / \mathrm{l}$ is 0.003185 . 
Follicular Phase

\section{LH / Allopregnanolone SC index}

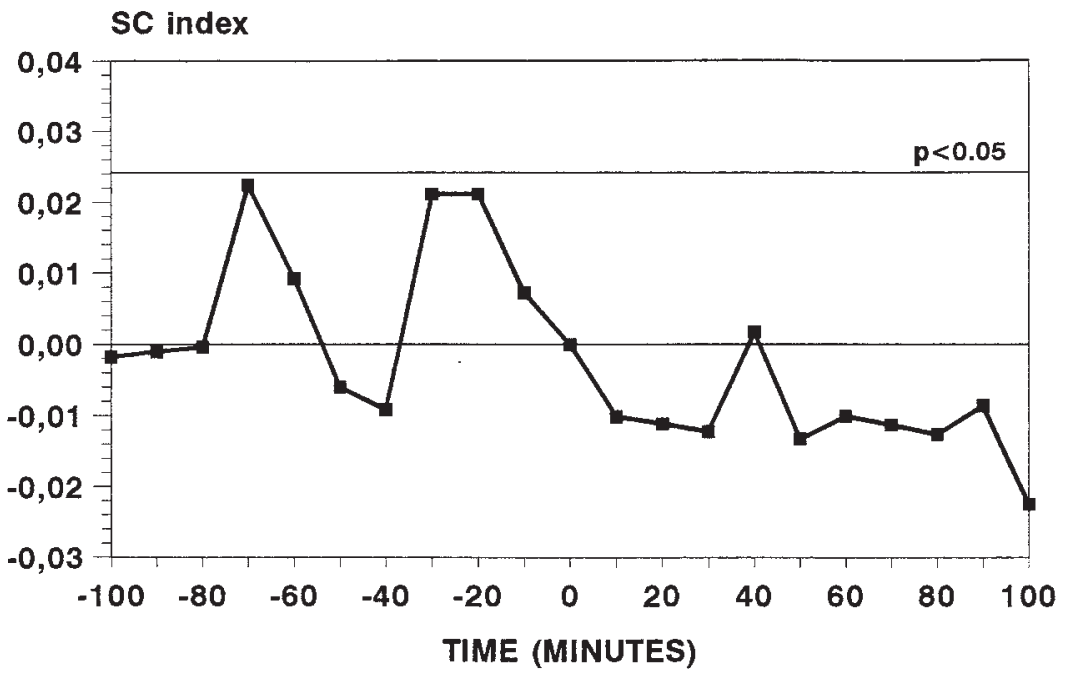

Luteal Phase

LH / Allopregnanolone SC index

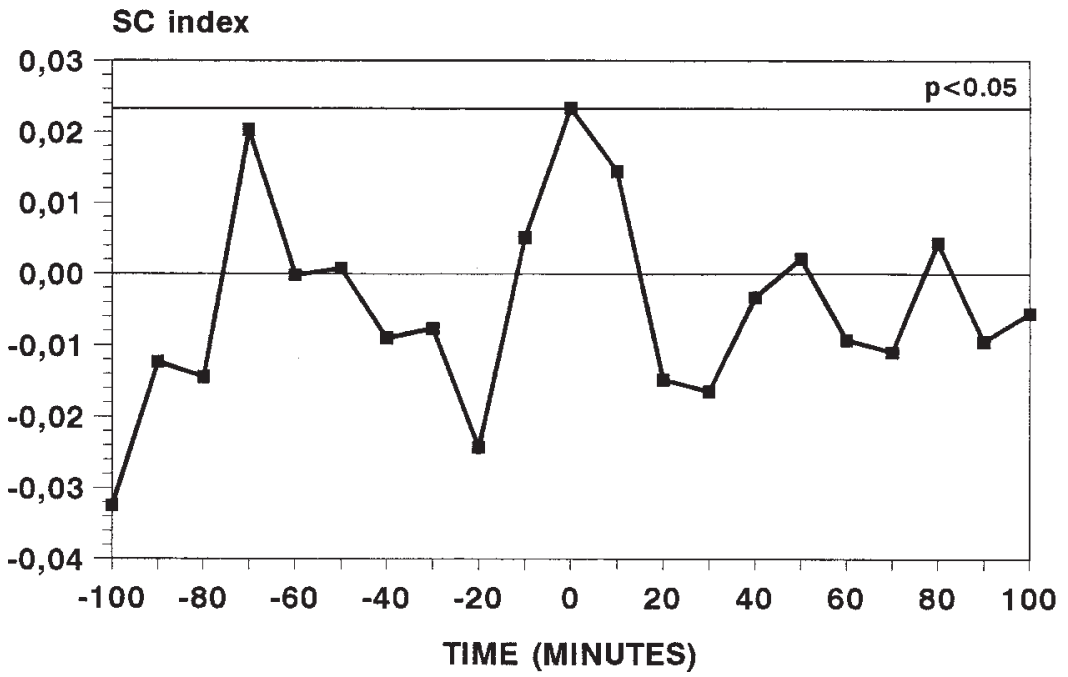

Figure 4 Mean specific concordance (SC) index between $\mathrm{LH}$ and allopregnanolone during the follicular (upper panel) and luteal phases (lower panel) of the menstrual cycle in one control subject. The SC index was significant only during the luteal phase at time 0 . synthesis. Indeed, this hypothesis is supported by previous data demonstrating that the maximum response to CRF bolus differ for cortisol and allopregnanolone, being after $15 \mathrm{~min}$ for cortisol and after $60 \mathrm{~min}$ for allopregnanolone (30). These delayed and distinct responses to $\mathrm{CRF}$ stimulation are in agreement with that observed for spontaneous secretory episodes, and confirm the time lag between the onset of cortisol pulses (taken as a marker of CRF-ACTH secretion) and the onset of allopregnanolone pulses. The fact that, in both eumenorrheic and amenorrheic subjects, cortisol showed an additional coupling time lag around the $+70 /+80$ interval, might be ascribed to a periodicity in the occurrence of both cortisol and allopregnanolone pulses. The knowledge of the physiological pattern(s) of the neuroactive steroid allopregnanolone is of great relevance and may allow correction of an unbalanced allopregnanolone production/secretion with appropriate 
Follicular Phase

Cortisol / Allopregnanolone SC index

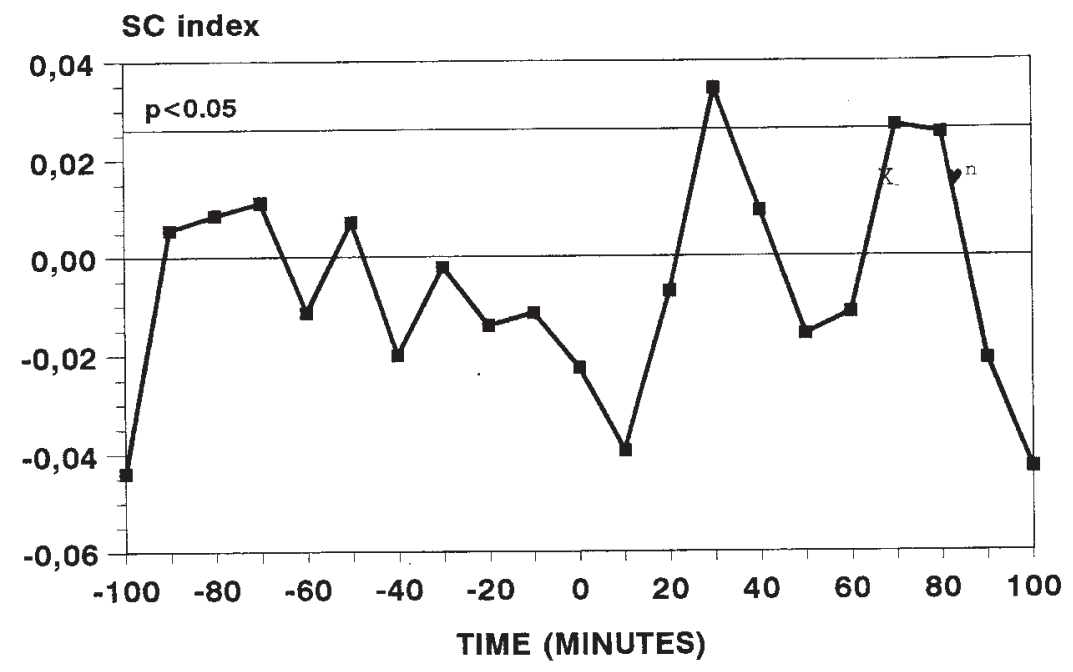

Luteal Phase

Cortisol / Allopregnanolone SC index

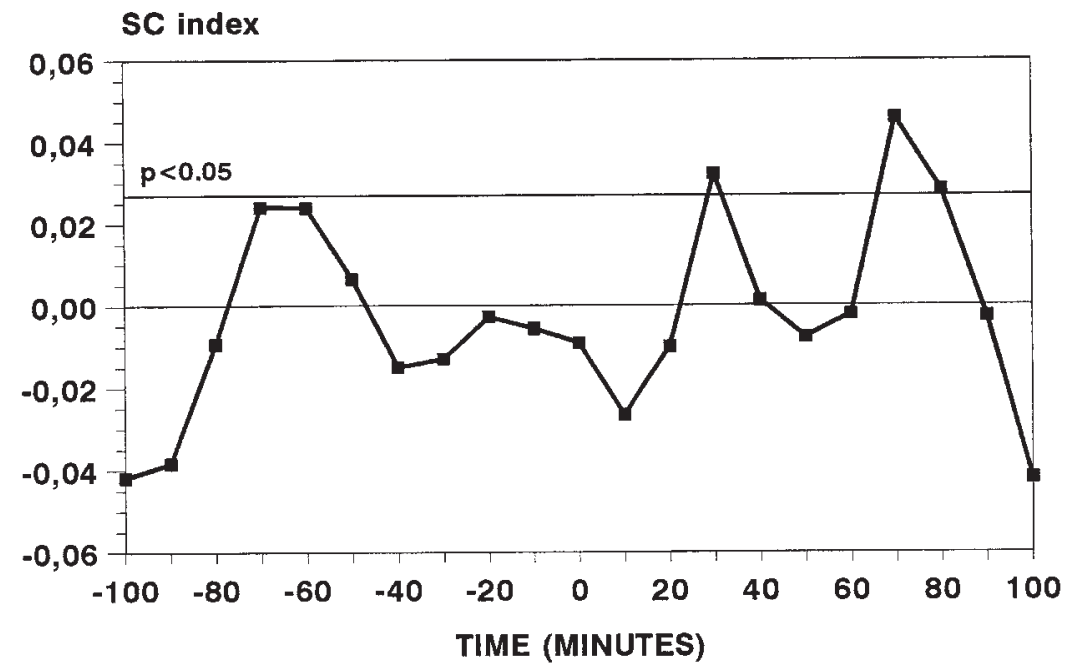

Figure 5 Mean SC index between cortisol and allopregnanolone during the follicular (upper panel) and luteal phases (lower panel) of the menstrual cycle in one control subject. The SC index was significant at times +30 and $+70 /+80 \mathrm{~min}$. therapeutic intervention. In fact, most of the changes in allopregnanolone concentrations observed in some physiopathological conditions, such as PMS (13), menopause (7) or aging (7), are probably related to the modification(s) of the spontaneous episodic release of allopregnanolone from the ovary and/or adrenal glands, mainly due to changes in its production and/or secretory mechanisms. The latter are typical in aging and in postmenopausal women (7).
In conclusion, the present study demonstrated that allopregnanolone is episodically secreted during fertile life as well as in stress-induced hypothalamic amenorrhea. In addition, a specific temporal coupling links LH to ovarian allopregnanolone production during the luteal phase of the cycle, while ACTH/cortisol secretion is coupled with allopregnanolone production during both phases of the cycle as well as in hypothalamic amenorrhea. 
SC index

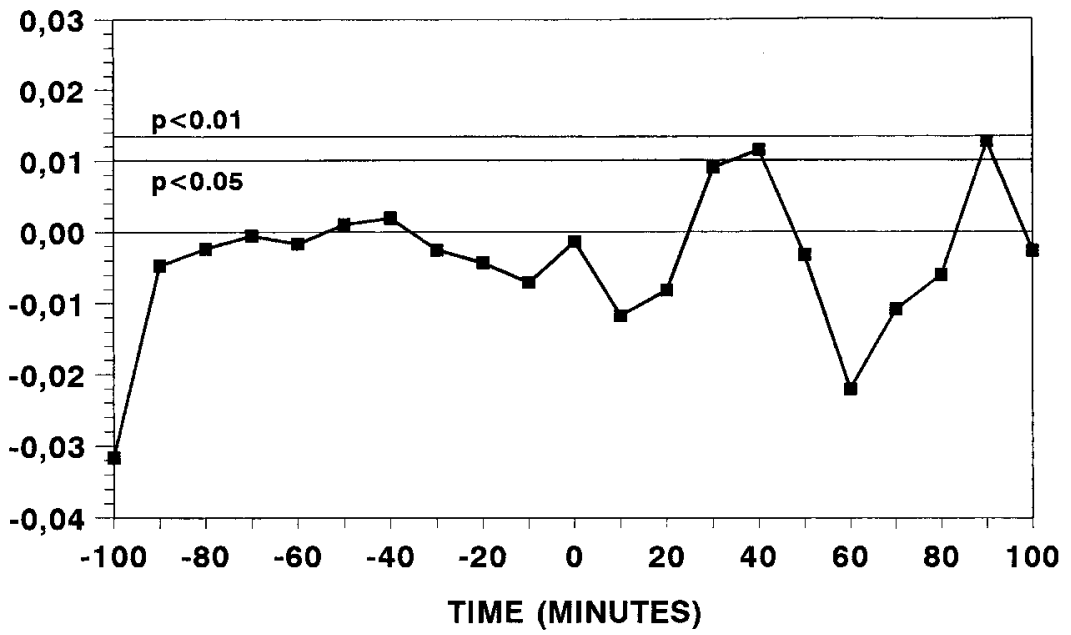

Figure 6 Mean SC index between cortisol and allopregnanolone in amenorrheic subjects. The SC index was significant at times +40 and +90 min and was very similar to that in eumenorrheic subjects.

\section{Acknowledgements}

We are grateful to Dr R H Purdy (Department of Psychiatry, Veterans Administration Hospital, San Diego, California, USA) for providing us with polyclonal antisera raised against allopregnanolone and to Dr David Rodbard (Information Resources Outreach and Liaison Activities, Association American Medical Colleges, Washington, DC, USA) for his helpful criticism. We are also grateful to Dr Patrizia Monteleone for editorial revisions.

\section{References}

1 Paul SM \& Purdy RH. Neuroactive steroids. FASEB Journal 1992 $62311-2322$.

2 Mellon SH \& Deschepper CF. Neurosteroid biosynthesis: genes for adrenal steroidogenic enzymes are expressed in the brain. Brain Research $1993629283-292$.

3 Robel P \& Baulieu EE. Neurosteroids: biosynthesis and function. Trends in Endocrinology and Metabolism 19945 1-8.

4 Majewska MD. Neurosteroids: endogenous bimodal modulators of GABA-A receptor. echanism of action and physiological significance. Progress in Neurobiology 199238 379-395.

5 Baulieu EE, Schumaker M, Koenig H, Jung-Testas I \& Akwa Y. Progesterone as a neurosteroid: actions within the nervous system. Cellular and Molecular Neurobiology 199616 143-147.

6 Baulieu EE \& Robel P. Neurosteroids: a new brain function? Journal of Steroid Biochemistry and Molecular Biology 199037 395-403.

7 Genazzani AR, Petraglia F, Bernardi F, Casarosa E, Salvestroni C, Tonetti A, Nappi RE, Luisi S, Palumbo M, Purdy RH \& Luisi M. Circulating levels of allopregnanolone in humans: gender, age, and endocrine influences. Journal of Clinical Endocrinology and Metabolism 199883 2099-2103.

8 Corpechot C, Young J, Calvel M, Wehrey C, Veltz JN, Touyer G, Mouren M, Prasad VV, Banner C, Sjovall J et al. Neurosteroids: 3 alpha-hydroxy-5 alpha-pregnan-20-one and its precursor in the brain, plasma, and steroidogenic glands of male and female rats. Endocrinology $19931331003-1009$.

9 Genazzani AR, Palumbo MA, de-Micheroux AA, Artini PG, Criscuolo M, Ficarra G, Guo AL, Benelli A, Bertolini A \& Petraglia F. Evidence for a role for the neurosteroid allopregnanolone in the modulation of reproductive function in female rats. European Journal of Endocrinology 1995133 375-380.

10 Barbaccia ML, Roscetti G, Trabucchi M, Purdy RH, Mostallino MC, Concas A \& Biggio G. The effects of inhibitors of GABAergic transmission and stress on brain and serum allopregnanolone concentrations. British Journal of Pharmacology 1997120 $1582-1588$.

11 Palumbo MA, Salvestroni C, Gallo R, Guo AL, Genazzani AD, Artini PG, Petraglia F \& Genazzani AR. Allopregnanolone concentrations in hippocampus of prepubertal rats and female rats throughout estrous cycle. Journal of Endocrinological Investigation 199518 853-856.

12 Genazzani AR, Salvestroni C, Guo AL, Palumbo M, Cela V, Casarosa E, Luisi M, Genazzani AD \& Petraglia F. Neurosteroids and regulation of neuroendocrine function. In The brain: source and target for sex steroid hormones, pp 83-91. Eds AR Genazzani, F Petraglia \& RH Purdy. New York: Parthenon Publishing, 1996.

13 Monteleone P, Luisi S, Tonetti A, Bernardi F, Genazzani AD, Luisi M, Petraglia F \& Genazzani AR. Allopregnanolone concentrations and premenstrual syndrome. European Journal of Endocrinology 2000142 269-273.

14 Wang M, Seippel L, Purdy RH \& Backstrom P. Relationship between symptom severity and steroid variation in women with premestrual syndrome: study on serum pregnanolone sulfate, 5-pregnan-3,20-dione and 3-hydroxy-5-pregnan-20-one. Journal of Clinical Endocrinology and Metabolism 199681 1076-1082.

15 Genazzani AR, Petraglia F, Monzani A, Alessandrini G \& Volpe A. Stress and reproduction. In Advances in Gynecological Endocrinology, pp 119-124. Eds AR Genazzani, F Petraglia, A Volpe \& F Facchinetti. London, UK: Parthenon Publishing, 1988.

16 Berga SL, Mortola SF, Girton L, Suh B, Laughlin G, Pham P \& Yen SSC. Neuroendocrine aberrations in women with functional hypothalamic amenorrhea. Journal of Clinical Endocrinology and Metabolism 198968 301-308.

17 Giles DE \& Berga SL. Cognitive and psychiatric correlates of functional hypothalamic amenorrhea: a controlled comparison. Fertility and Sterility $199360486-492$.

18 American Psychiatric Association. Diagnostic and statistical manual of mental disorders, edn 4. Washington, DC: American Psychiatric Association, 1995.

19 Genazzani AD, Petraglia F, Fabbri G, Monzani A, Montanini V \& Genazzani AR. Evidence of luteinizing hormone secretion in hypothalamic amenorrhea associated with weight loss. Fertility and Sterility $1990 \mathbf{5 4} 222-226$.

20 Genazzani AD, Petraglia F, Benatti R, Montanini V, Algeri I, Volpe A \& Genazzani AR. Luteinizing hormone (LH) secretory burst duration is independent from LH, prolactin, or gonadal steroid 
plasma levels in amenorrheic women. Journal of Clinical Endocrinology and Metabolism 199172 1220-1225.

21 Genazzani AD, Gamba O \& Petraglia F. Estrogen replacement therapy modulates spontaneous $\mathrm{GH}$ secretion but does not affect $\mathrm{GH}-\mathrm{RH}$-induced $\mathrm{GH}$ response and low $\mathrm{T} 3$ syndrome in women with hypothalamic amenorrhea associated to weightloss. Journal of Endocrinological Investigation 199821 353-357.

22 Oerter KE, Guardabasso V \& Rodbard D. Detection and characterization of peaks and estimation of instantaneous secretory rate for episodic pulsatile hormone secretion. Computers and Biomedical Research 198619 170-191.

23 Genazzani AD. Application of peak-detection programs to clinical data. In Computers in Endocrinology: Recent Advances, pp 71-82. Eds V Guardabasso, D Rodbard \& G Forti. New York: Raven Press, 1990.

24 Genazzani AD \& Rodbard D. Evaluation of methods for detection of pulsatile hormone secretion: sensitivity vs specificity. Acta Endocrinologica 1991124 295-306.

25 Guardabasso V, Genazzani AD, Veldhuis JD \& Rodbard D. Objective assessment of concordance of secretory events in two endocrine time series. Acta Endocrinologica $1991 \mathbf{1 2 4} 208-218$.

26 Genazzani AD, Petraglia F, Pianazzi F, Volpogni C \& Genazzani AR. The concomitant release of androstenedione with cortisol and luteinizing hormone pulsatile releases distinguishes adrenal from ovarian hyperandrogenism. Gynecology and Endocrinology $1993733-41$
27 Nappi RE, Petraglia F, D’Ambrogio G, Zara C \& Genazzani AR. Hypothalamic amenorrhea: evidence of a central derangement of hypothalamic-pituitary-adrenal cortex axis activity. Fertility and Sterility $199359571-576$.

28 Genazzani AD, Petraglia F, Volpe A \& Genazzani AR. Hypothalamic amenorrhea: neuroendocrine mechanisms/stress-induced anomalies. Assisted Reproductive Technology/Andrology 19979 $1-13$.

29 Genazzani AD, Guardabasso V, Petraglia F \& Genazzani AR. Specific concordance index defines the physiological lag between $\mathrm{LH}$ and progesterone in women during the midluteal phase of the menstrual cycle. Gynecology and Endocrinology $1991 \mathbf{5}$ $175-184$.

30 Bernardi F, Lanzone A, Cento RM, Spada RS, Pezzani I, Genazzani AD, Luisi S, Luisi M, Petraglia F \& Genazzani AR. Allopregnanolone and dehydroepiandrosterone response to corticotropinreleasing factor in patients suffering from Alzheimer's disease and vascular dementia. European Journal of Endocrinology 2000 $142466-471$.

Received 16 July 2001

Accepted 9 November 2001 